(C) 2011 IEEE. Reprinted, with permission, from Ball, L., Bradley, D., Szymkowiak, A. and Brownsell, S. Linking recorded data with emotive and adaptive computing in an eHealth environment. 1st IEEE Conference on Healthcare Informatics, Imaging and Systems Biology, San Jose, California, 26-29 July, 2011.

This material is posted here with permission of the IEEE. Such permission of the IEEE does not in any way imply IEEE endorsement of any of University of Abertay Dundee's products or services. Internal or personal use of this material is permitted. However, permission to reprint/republish this material for advertising or promotional purposes or for creating new collective works for resale or redistribution must be obtained from the IEEE by writing to pubspermissionsdieee.org.

By choosing to view this document, you agree to all provisions of the copyright laws protecting it. 


\section{Linking Recorded Data with Emotive and Adaptive Computing in an eHealth Environment}

\author{
Leslie Ball, David Bradley, Andrea Szymkowiak \\ Computing \& Engineering Systems \\ University of Abertay \\ Dundee DD1 1HG, United Kingdom \\ 1.ball@abertay.ac.uk,d.bradley@abertay.ac.uk \\ a.szymkowiak@abertay.ac.uk
}

\author{
Simon Brownsell \\ School of Health and Related Research \\ University of Sheffield \\ Sheffield S1 4DA, United Kingdom \\ s.brownsell@sheffield.ac.uk
}

\begin{abstract}
Telecare, and particularly lifestyle monitoring, currently relies on the ability to detect and respond to changes in individual behaviour using data derived from sensors around the home. This means that a significant aspect of behaviour, that of an individuals emotional state, is not accounted for in reaching a conclusion as to the form of response required. The linked concepts of emotive and adaptive computing offer an opportunity to include information about emotional state and the paper considers how current developments in this area have the potential to be integrated within telecare and other areas of eHealth. In doing so, it looks at the development of and current state of the art of both emotive and adaptive computing, including its conceptual background, and places them into an overall eHealth context for application and development.
\end{abstract}

Keywords-emotive computing, adaptive computing, telecare, eHelath, lifestyle monitoring

\section{INTRODUCTION}

A sub-set of a wider and more general model of telecare, lifestyle monitoring systems set out to provide information on an individual's pattern of behaviour to support a mode of operation which supports earlier, and hence preventative, care interventions through the identification of behaviour change. This is in contrast with responsive approach that is currently used in which historic data is used to assess individual need. However, despite a continuing interest in the precepts of lifestyle monitoring following the study undertaken by Celler et al. [1] in 1995, which concluded that some $50 \%$ of individuals had undiagnosed medical problems that could be detected by home monitoring, the formal evidence base for this form of telecare remains weak. This despite the significant numbers of commercial installations worldwide.

A recent literature review involving the authors [2] identified that current commercial lifestyle monitoring systems rely for their operation on the deployment and use of a range of sensors, the most common of which are set out in Table I, to provide a profile of an individual's behaviour. This profile is then currently interpreted, generally retrospectively, to provide an estimate of the monitored individual's state of health.

However, at present, and despite continuing efforts to deploy the methods and approaches of artificial intelligence and machine learning to isolate and identify abnormal patterns of behaviour or changes to established behaviours, the algorithms thus far employed tend to be far from robust and emphasise general levels of activity rather than detailed patterns of behaviour $[3,4,5,6,7,8,9]$. Further, the sensors used are focused on the physical environment within which the individual functions, in most instances their home, and fails to take into account of other factors, as for instance emotional state of the individual.

Additionally, previous research involving certain of the authors and structured around literature searches and focus groups identified a range of indicators which were linked to changes in an individual's status, and hence in their care needs [10]. These indicators are set out in Table II and suggest that there is a significant, but as yet unaddressed, observed and recorded component associated with many of these indicators which represents an important, and as yet untapped, resource. At present, the primary sources for any

TABLE I. PRIMARY LIFESTYLE MONITORING SENSOR TECHNOLOGIES

\begin{tabular}{|l|l|l|l|}
\hline Sensor or Monitored Parameter & Temperature & Door open/close & Humidity \\
\hline PIR & Lights & Sink/Bath use & Telephone \\
\hline Drawer and cupboard use & TV and video use & Flood & Bed/Chair occupancy \\
\hline General electrical appliance use & Gas (Flame sensor) & & \\
\hline Fall detection & & \\
\hline
\end{tabular}


TABLE II. RELATIONSHIP BETWEEN SELECTED STATUS INDICATORS, POSSIBLE MEASURES FOR THOSE INDICATORS AND THE ASSOCIATED DATA SOURCES

\begin{tabular}{|c|c|c|c|}
\hline Indicator & & Data Source & \\
\hline Decline in general personal care/ADL & - Observation & - Activity (Room PIRs) & - Chair/bed occupancy \\
\hline Change in time spent at home & - Activity (Room PIRs) & & \\
\hline Change in sleeping patterns & - Activity (Room PIRs) & - Bed sensor & \\
\hline Change in amount of food eaten & $\begin{array}{l}\text { - Observation } \\
\text { - Appliance sensors }\end{array}$ & - Activity (Room PIRs) & - Cupboard/refrigerator use) \\
\hline Change in food storage & - Observation & - Cupboard/refrigerator use & \\
\hline Change in preparing meals & - Activity (Room PIRs) & - Cupboard/refrigerator use & - Appliance use \\
\hline Changes in Washing/Bathing & - Activity (Room PIRs) & & \\
\hline Falls/falling & - Observation & - Activity (Room PIRs) & \\
\hline Change in visitor numbers & - Observation & - Activity (Room PIRs) & - Chair/bed occupancy \\
\hline Dirty pots & - Observation & - Activity (Room PIRs) & \\
\hline Change in contact with healthcare services & - Observation & - Activity (Room PIRs) & \\
\hline General neglect/poor state of household & - Observation & & \\
\hline Presence of smells or odours & - Observation & & \\
\hline
\end{tabular}

such recorded data would be carers, family and friends, health visitors and general visitors, the majority of whom are not trained either in observation or reporting, yet whose observations have the potential to be a significant contributor to the interpretation of the data provided by sensors and sensing systems structured around the physical environment. The ability to capture and record such data is therefore likely to be a significant contributor to enhancing the effectiveness of lifestyle reassurance and monitoring systems, and hence its acceptance.

Such data would also be expected to include, or be influenced by, the observer's subjective assessment of the emotional state of the individual concerned. An ability to provide an independent assessment of an individual's emotional state therefore has the possibility of adding considerably to the performance of a lifestyle monitoring system through the integration of an understanding of that state with the sensory data.

In this context, while lifestyle monitoring systems have the potential to provide an effective reference as to an individual's physical activity, they provide little evidence of their emotional state. Recent years have seen significant development in what is referred to as emotive or affective computing systems $[11,12,13,14]$ that recognize, interpret, process, simulate and respond to human emotions with the goal of interpreting the emotional state of the individual and hence adapting their behaviours in response $[15,16,17]$.

Thus as an supplement to reliance on what might be considered as a relatively ad hoc form of data collection in the recording of observer related data, and an assessment of emotional state based on that observation, it is suggested that an opportunity exists to exploit developments in emotive computing to supplement both the sensor and the recorded data by evaluating the way in which an individual carries out a task or tasks within the home and to use this to detect relatively subtle changes in their emotional state, and hence their behaviour.

Developments in this area are now beginning to be seen, as for instance in the implementation of emotional avatars in Dementia care [18], technology aimed at enhancing social inclusion for the elderly in their homes through the deployment of an affective state recognition system integrated with an interactive web-based system to communicate with family and friends [19] and wearable systems aimed at detecting emotional state [20].

In the paper, following an overview of the aims of lifestyle monitoring and the role of observation together with a brief summary of the conceptual development of emotive computing, consideration is given to their potential applications within lifestyle monitoring with particular reference to a framework for development and deployment. In this context therefore the paper builds on and develops the case for considering the introduction of emotive and adaptive computing in telecare previously put forward in a short editorial format [21].

\section{REVIEW OF LITERATURE}

A preliminary review of literature identified a total of some 290 papers and articles which were grouped into the sub-categories shown in Table III. Of these, less than $5 \%$ referred directly to applications in eHealth.

While this is not, and was not intended to be, a formal systematic review, it nevertheless provides a very useful indicator as to the direction of current work in this area, which is focussed on methods and applications covering a number of domains ranging from health to robotic communication and security as well as a generic understanding of human, and indeed machine, behaviour. 
TABLE III. LITERATURE REVIEW CLASSIFICATION

\begin{tabular}{|c|c|c|c|}
\hline Category & No & Category & No \\
\hline Acceptance of technology & 40 & Activity measures & 8 \\
\hline Baby Boomers & 15 & Behaviour & 24 \\
\hline Cognition & 1 & Decision support & 15 \\
\hline Diagnostics & 28 & Emotion/Emotive & 8 \\
\hline Other & 70 & Gesture & 13 \\
\hline Models & 5 & Monitoring & 25 \\
\hline Questionnaires & 8 & Resources & 3 \\
\hline Standards & 10 & Support systems & 17 \\
\hline \multicolumn{4}{|c|}{ Total papers $=290$} \\
\hline
\end{tabular}

\section{THE AIM OF LIFESTYLE MONITORING AND THE ROLE OF OBSERVATION}

The aims of lifestyle monitoring in supporting individuals in living independently can be summarized as follows:

1. Ensuring reliable and effective data systems and structures linked to a clearer understanding of the needs of users. This also implies the ability to integrate new types of data, such as recorded and emotional data.

2. Provide timely and effective interventions such that changes in behaviour, indicative of the need for a specific health or care intervention or need for a different intensity of service, can be detected and responded to earlier than is at present the case.

3. Predict individual and population based needs. By isolating and identifying patterns of activity and behaviour associated with an individual's wellbeing and comparing these with the data generated by other individuals in similar circumstances, it becomes possible to isolate and identify similar patterns, thus predicting future need and the possibility to mitigate against this.

4. Provide real-time health and care assessments which can be integrated into current service models in combination with traditional assessments to inform care provision.

In this context, as can by seen by reference to Table 2 , observation can provide additional information on a wide range of behavioural related issues. However, as has already been suggested, such recorded data can be associated with a wide range of sources with different recording skills and focus. Potential strategies for gathering this information include the use of structured questionnaires, as for instance those currently available for assessing an individuals selfperception or activities of daily living [22,23,24], and the free-form analysis of text based around natural language processing, perhaps linked to the users mobile phone for both the collection and onward transmission of the data.

\section{EMOTIONAL INTELLIGENCE}

Current research by the authors is considering applications of adaptive and emotive computing in relation to cybercrime and the associated counter-measures. This is a particularly significant area given the increased numbers of both terrorist and criminal cells operating in this area and the consequent threats they pose to governments, society and commercial infrastructure. These threats have recently received the highest UK government priority in terms of national security and defence budgets [25].

As an illustration of one of the approaches in this field being considered in relation to emotive computing, consider the example of ubiquitous keyboard usage. Here, new biometric methods are being developed to identify individuals and assess their behaviours based on their interactions with those technologies. Previous research has already considered identification of users from their natural 'short' typing signature [26] and also from freeform textual patterns [27]. This potential ability to detect and react to emotional states establishes and defines research questions as to whether it is possible to not only identify an individual, but also to ascertain their stress level either at the time of using the keyboard or in a post-event analysis.

The approach has ramifications for providing an additional layer of security for password protected logons. For example, an easy way to breach cyber-security is to observe (or record) someone typing in their username and password. A person attempting this type of identity theft should not be able to penetrate the security as research theory and practice is consolidating that a typing signature is unique and therefore an impostor should have difficulty mimicking a valid user's typing rhythm. This is the digital equivalent to a handwritten signature. It is this potential ability to detect and react to emotional state which underpins the present paper and the transference of the underlying research concepts and achieved outcomes from the domain of security to that of teleheath, in particular telecare and lifestyle monitoring.

\section{A. Link to psychology}

Issues of digital emotive detection lie at the interface between computer science and experimental psychology. Current experimental work involving the authors is based on the use of galvanic skin response (GSR) systems to measure the effect of different levels of stress on an individuals typing signature in an attempt to identify whether an emotive signature can be identified for stress. This could then potentially be used to raise a security alert to monitoring networks and cyber-security infrastructures which would, for instance, indicate that the individual was operating under duress [28].

It is also worth noting in the context of the paper that one of the responses to a discussion of certain of the authors work that appeared in the magazine New Scientist [29] also identified a potential link to eHealth applications with the suggestion that a typing signature could in some way be mapped to recognise the onset or deterioration of a particular disease. The same potential link was also recognised in a later article in the same magazine [30].

The ubiquitous nature of keyboard usage certainly lends itself to non-intrusive and regular data capture from anyone in patient-care and could, for instance, be of particular use monitoring out-patients or individuals post discharge from hospital. 


\section{B. Biometrics and motor control}

Biometric research focuses on the identification of individuals using physiological (e.g. finger printing and retinal analysis) and behavioural characteristics (e.g. writing, typing, walking, facial expressions and so forth). The idea of a 'generalised motor program' [31] suggests that movements, in combination with an individual's anatomical characteristics, show regular patterns which can be used for identifying an individual. A motor program, in which only force and timing parameters are adjusted to produce a particular motor output, will result in equivalent movement patterns. For example, an individual's rate of handwriting can be fast or slow, but the pattern of the signature will be very similar.

The assumption of a motor program suggests that motor behaviour is repeatable and thus may be the basis for biometrics applications in which individuals are recognised via their motor behaviour. The regulation of force and timing parameters a parameters of a motor program are also relevant when typing [32]. More specifically, timing patterns of inter-key presses and key hold times result in reliable identification and authentication of individuals $[27,33]$.

In an age where more and more users have access to a computer or technological system that requires them to enter password, usernames or PIN numbers, the usage of keyboard entries will become increasingly more common. Thus, it is possible to anticipate that the coming 'older' generations will have considerable experience in using keyboard based devices in their homes as well in the future, not to mention being exposed to a variety of other ubiquitous digital technologies.

\section{Health biometrics}

There is substantial potential for investigating motor behaviour as a biometric, and in particular for health applications. The ubiquitous use of digital technology raises the question of whether biometric systems, such as those based on - for example, but naturally not limited to - typing sequences can be used in order to identify individuals with the early stages of dementia or Parkinson Disease. There is evidence that shows that motor control (e.g. finger tapping) is impaired in individuals with early stage Parkinson Disease [34] when compared to a healthy elderly control group.

A number of other motor behaviours can be affected by conditions associated with aging, such as dementia [35], and eye movements in individuals with dementia and Parkinson's [36] to identify but a few studies. Thus, a biometric system that can compare an individual's change in motor behaviour over time could potentially be very useful as a diagnostic tool, especially if it is unobtrusive, as many telehealth and eHealth systems are. In addition, biometric systems that can tap into the emotions of individuals analysing facial expression [37,38], could have an enormous potential to identify individuals in distress, which again could be of crucial relevance in a Health-related environment. Future research will show to which extent these systems can be usefully applied in the areas of eHealth and be accepted by a user.

\section{EMOTIVE COMPUTING AND LIFESTYLE MONITORING}

As already stated, the aim of a lifestyle monitoring system approach is to provide an interpretation of individual behaviour such that changes in that behaviour can be linked to a change in care needs, enabling the appropriate response. Within this context an ability to detect, and respond to, the emotional state of the individual has significant potential, in particular when linked to the recording of a range and types of recorded data. This leads in turn to the consideration of a number of areas in which emotive computing could be deployed.

\section{A. Speech}

Emotional speech processing establishes emotional state by analyzing speech patterns using vocal parameters and prosody features with pitch variables and speech rate analyzed through the use of pattern recognition $[39,40,41,42]$. Within a lifestyle monitoring context, the application of such analysis to established forms of communication, as for instance in response to a warden call or a telephone conversation with a carer, could provide information which would then be used to supplement the data recorded by those same individuals, as for instance following a visit.

There is the further possibility that certain specific types of illness, as for instance a cold or longer term conditions, which might effect behaviour could be identified, enabling an appropriate, and early response to prevent a possible escalation of the condition to a point where it requires a more major intervention.

\section{B. Facial expression}

The detection and processing of facial expression is achieved through the use of a range of methods and techniques, as for instance as optical flow, hidden Markov models, neural network processing or active appearance models. In this context, it is likely that there will be a requirement to integrate a number of different modalities, as for instance facial expression and speech prosody [43] or facial expressions and hand gestures [44] in order to provide an effective and robust estimation of the subject's emotional state.

Given that evidence gather by the authors on individual views on the use of video monitoring suggests that accessing visual information of the required form may be more difficult than for speech, the increased use of video cameras for communication with, family and friends may provide a potential source. There are however significant acceptance and ethical issues that must be addressed before such use becomes either possible or practical.

\section{Gestures}

Body language, and particular gestures [45,46], are significant issues in expressing emotional state, and a change in either of these areas can be indicative of an individuals current emotional state. In this context, 
significant research effort has been deployed to date into the recognition of hand gestures, particularly in relation to gaming and games technologies $[47,48,49]$.

Within the context of lifestyle monitoring, the capture of gestures presents similar problems and issues to those associated with the capturing facial expression in the need to provide a camera, something which lifestyle monitoring users have, as already suggested, not generally been receptive to.

\section{Physiological monitoring}

The development of a range of worn sensors for physiological parameters such as heart rate, temperature and movement leads to considerations of the possibility of using these in relation to aspects of emotive computing [50,51,52]. This then supports the potential integration of such physiological data with more conventional data forms associated with telecare, including other sources of emotion related data.

\section{E. Integeration with recorded data}

Recorded data can be used to record both factual data, as for instance the quantities of dishes in a sink or the responses to specific questions, as well as more subjective data which expresses the observer's subjective interpretation of the emotional state of an individual as detected in, say, conversation. While such recorded data is believed to be of significant potential in facilitating the interpretation of behaviour, its very subjectivity leads suggests that it lacks robustness. If this subjective data could be supplemented and reinforced by objective data derived from the use of adaptive and emotive computing strategies, its value would be significantly enhanced.

Thus, the recorded data may suggest that an individual may be angry could be supplemented by information from voice analysis suggesting raised levels of stress in that individual. Such linkage is at present speculative, but if capable of being realised offers significant potential.

\section{F. Links to mobile telecare}

Present lifestyle monitoring and telecare systems are tied to the users home environment and there is a requirement to extend aspect of the support such system provide into the wider domain. The development of mobile technologies provides the opportunity to consider means by which such an extension might be achieved, including the transference of novel forms of information gathering, perhaps linked to adaptive and emotive computing through features such as route analysis or system use.

\section{G. System structure and use}

The potential to detect behavioural change through the deployment and use of a range of both objective and subjective strategies has significant potential for telecare. Thus, keyboard use could be used to support the identification of an individual along with their emotional state, as for instance through the recording of the force and speed with which both individual and specific keys on a keyboard are pressed. Similarly, analysis of voice or gesture could be used to add to information gained by observation to enhance the quality of the outcomes of the analysis of that data.

Further, this suggests that in addition to an increased ability to interpret behaviour in relation to the requirements of lifestyle monitoring, there is an as yet unconsidered potential to detect and respond to a range of conditions such as dementia and Parkinson's disease, along with potentially more subtle behavioural changes such as those that would be associated with the indicators set out in Table 2 above [53].

That these developments are possible is supported by a consideration of those generations who will be moving to retirement in the immediate future. Unlike their predecessors, these next generations will increasingly have backgrounds which involves the regular use of computers, and hence of keyboards, as well as gaming consoles. Factors which are already in evidence in the continuing growth in the number of 'silver surfers' and developments such as the University of the Third Age [54,55,56,57].

Such generations of users might also be expected both to demand technology to support hem in living independently, as well as being more willing to accept such technology. All of which affords opportunities to integrate based means of emotional analysis, linked to emotive computing, into lifestyle monitoring. Figure 1 therefore suggest, in much simplified form, a potential model of the information flows that might be associated with the implementation of a lifestyle monitoring system based around the above precepts.

\section{SYSTEM DEVELOPMENT}

The authors are currently seeking to build on their work in both telecare and emotive computing to implement the ideas and concepts set out in the paper. Specifically, their immediate aims are:

- To extend the existing literature review as reported in the paper into a formal systematic review of work in this area.

- To carry out a full and formal study of the potential

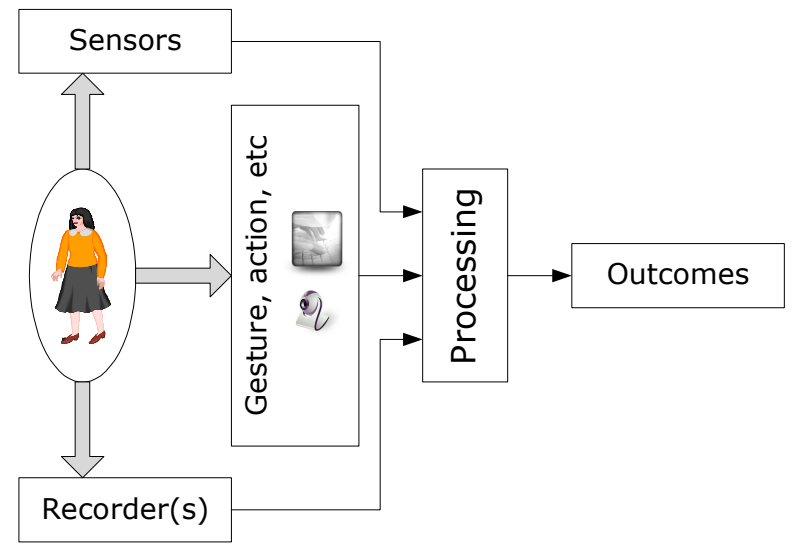

Figure 1. Basic, and much simplified, structure of information flows in telecare using a combination of sensors, recorded data and emotive computing. 
areas of application of both recorded data and adaptive and emotive computing within both telecare and lifestyle monitoring.

- To seek to establish user attitudes to the potential range of technologies and the likely levels of acceptance.

- To establish and prioritise areas for development in the context of both the technology and user need while taking account of strategic developments in healthcare and associated issues.

- To define active lines of development at the prototype stage through consideration of both technological and human issues.

The above are also intended to form the basis of the questions to be associated with a research programme which will seek to develop the concept of emotive computing as applied to eHealth in general, and to aspects of telecare and lifestyle monitoring in particular.

\section{CONCLUSIONS}

While the applications of observation and emotive or adaptive computing within lifestyle monitoring are currently sparse, it is believed that there are significant opportunities to take advantage of changes both in technology and attitudes that are likely to take place in coming years. Specifically, the significantly increased familiarity with and use of computers by coming generations older people will enable the elements of emotive computing to be integrated with features such as keyboard use and the use of web cams to provide additional information on emotional state. This data, when integrated with data from the more conventional sensors associated with lifestyle monitoring as well as with recorded data is believed to afford significant opportunities for system enhancement and the identification of changes in user status, and hence of need.

In the context of the paper, the emphasis has been on establishing that there is a role both for observation and adaptive and emotive computing within lifestyle monitoring and telecare in particular, while acknowledging that there are potentially wider issues, as for instance the detection of specific conditions, that also need to be considered. Indeed, it is believed that there is considerable potential for much wider, and more general, applications within healthcare. For instance, could facial recognition systems linked to emotive computing to assess degrees of discomfort be used in support of remote therapies to improve the quality, safety and effectiveness of such therapies or could Parkinson's disease be monitored through the monitoring of speech and hand tremor?

Finally, it should be the paper does not attempt to address the significant and important issue of health policy and the potential for the developments considered in the paper to impact on that policy. What is clear is that demographic changes are going to result in increasing numbers of older people, and that healthcare provision must adapt to accommodate this change. Lifestyle monitoring and telecare are of themselves just one aspect of the changes that are being proposed as part of this change in provision.

\section{REFERENCES}

[1] Celler BG, Earnshaw W, Ilsar ED, Betbeder-Matibet L, Harris MF, Clark R, Hesketh T and Lovell NH, "Remote monitoring of health status of the elderly at home. A multidisciplinary project on aging at the University of New South Wales", J. Biomedical Computing, 1995, 40(2): 147-55.

[2] Brownsell S, Bradley D, Blackburn S, Cardinaux F \& and Hawley S, "A Systematic Review of Lifestyle Monitoring Technologies", J. Telemedicine \& Telecare, 2010, 17;185 - 189

[3] Rahal Y, Mabilleau P and Pigot H, "Bayesian filtering and autonomous sensors for localization in a smart home", $21^{\text {st }}$ Intl. Conf. on Advanced Information Networking \& Applications, 2007, 2: 793 797

[4] Gopalratnam K and Cook DJ, "Online sequential prediction via incremental parsing: The active LeZi algorithm”, IEEE Trans. Intelligent Systems, 2007, 22(1): 52 - 58

[5] Vainio A, Valtonen M and Vanhala J, "Proactive Fuzzy Control and Adaptation Methods for Smart Homes", IEEE Trans. Intelligent Systems, 2008, 49(12): 1463 - 1469

[6] Virone G, Alwan M, Dalal S, Kell SW, Turner B, Stankovic JA and Felder, "Behavioural patterns in older adults in assisted living", IEEE Trans. Information Technology in Biomedicine, 2008, 12(3): 387 398

[7] Park S and Kautz H, "Hierarchical recognition of activities of daily living using multi-scale, multi-perspective vision and RFID", $4^{\text {th }}$ Intl. Conf. on Intelligent Environments, 2008, 1 - 4

[8] Luštrek M and Kaluža B, "Fall Detection and Activity Recognition with Machine Learning, Informatica", 2009, 33: 205-212

[9] Martínez-López R, Millán-Ruiz D, Martín-Domínguez A and ToroEscudero MA, "An Architecture for Next-Generation of Telecare Systems Using Ontologies, Rules Engines and Data Mining”, Intl Conf. on Computational Intelligence for Modelling, Control and Automation, 2008, 31-36

[10] Brownsell S, Aldred H, Hawley MS, "The role of telecare in supporting the needs of elderly people", J. Telemedicine and Telecare, 20078, 13: 293 - 297

[11] Picard RW, Affective Computing, MIT Press, 1997

[12] Picard RW, "Affective computing: challenges", Int. J. of HumanComputer Studies, 2003, 59(1/2): 55 - 64

[13] Jianhua Tao, Tieniu Tan and Picard RW (eds), Affective Computing and Intelligent Interaction, Proc. ${ }^{\text {st }}$ Int. Con. On Affective Computing \& Intelligent Interaction, Lecture Notes in Computer Science, Springer, 2005

[14] Hudlicka E, "To feel or not to feel: The role of affect in humancomputer interaction", Int. J. of Human-Computer Studies, 2003, 59(1/2): 1 - 32

[15] Breazeal C and Brooks R, "Robot emotion, a cultural perspective", 2005, robotic.media.mit.edu, 31 pages

[16] Fellous J-M, "From Human Emotions to Robot Emotions", American Association for Artificial Intelligence, 2004, 11 pages

[17] Picard RW, "Toward computers that recognize and respond to user emotion", IBM Systems Journal, 2000, 39(3/4): 705 - 719

[18] Wiratanaya A, Lyons M, Butko N and Abe S, "iMime: An Interactive Character Animation System for use in Dementia Care", Proc. $12^{\text {th }}$ Int. Conf. Intelligent User Interfaces, 2007: 262 - 265

[19] Peter C, Bieber G and Urban, B, "Affect- and Behaviour-Related Assistance for Families in the Home Environment", ACM, Proc. $3^{\text {rd }}$ Int. Conf. Pervasive Technologies Related to Assistive Environment, 2010, Samos, Greece

[20] Lisetti CL and Nasoz F, "Using noninvasive wearable computers to recognize human emotions from physiological signals", EURASIP J. on Applied Signal Processing, 2004, 11: 1672 - 1687

[21] Ball L, Bradley DA and Brownsell S, "Emotive computing may have a role in telecare", Invited editorial, 2011, J. Telemedicine \& Telecare, In print

[22] Benyaminia Y, Leventhalc EA and Leventha H, "Elderly people's ratings of the importance of health-related factors to their selfassessments of health", Social Science \& Medicine, 2003, 56;16611667 
[23] Keller HH, McKenzie JD and Goy RE, "Construct Validation and Test-Retest Reliability of the Seniors in the Community: Risk Evaluation for Eating and Nutrition Questionnaire", J. of Gerontology: MEDICAL SCIENCES,2001, 56A(9);M552 - M558

[24] Cumming RG, Salkeld G, Thomas M and Szonyi G, "Prospective Study of the Impact of Fear of Falling on Activities of Daily Living, SF-36 Scores, and Nursing Home Admission", J. of Gerontology: MEDICAL SCIENCES,2001, 55A(5);M299 - M305

[25] www.security-technologynews.com/news/uk-announces-nationalsecurity-strategy.html (Accessed on 9th February 2011)

[26] Bergadano F, Gunetti D and Picardi C, "User authentication through keystroke dynamics", ACM Transactions on Information and System Security, 2002, 5: 367-397

[27] Gunetti D and Picardi C, "Keystroke analysis of free text", ACM Transactions on Information Systems Security, 2005, 8: 312-347

[28] Dowman M, Szymkowiak A and Ball L, "A Biometric Security Method, System and Computer Program", International Patent Application No PCT/GB2010/050785

[29] Ball L, New Scientist

[30] New Scientist

[31] Summers JJ and Anson JG, "Current status of the motor program: Revisited", Human Movement Science 28, 2009, 566-577

[32] Viviani P and Terzuolo C, "On the relation between word-specific patterns and the central control model of typing: A reply to Gentner", J. Experimental Psychology: Human Perception and Performance, 8, 1982: 811-813

[33] Joyce R and Gupta G, "Identity authentication based on keystroke latencies", Communications of the ACM, 33, 1990: 168-176

[34] Goldman W, Baty J, Buckles V, Sahrmann S and Morris J, "Cognitive and motor functioning in Parkinson Disease: Subjects with and without questionable dementia", Archives of Neurology, 55 , 1998: 674-680

[35] Boyle P, Cohen R, Paul R, Moser D and Gordon N, "Cognitive and motor impairments predict functional declines in patients with vascular dementia”, Intl. J.1 of Geriatric Psychiatry, 47, 2002: 164169

[36] Bronstein AM and Kennard C, "Predictive ocular control in Parkinson's Disease", Brain, 108(4), 1985: 925 - 940

[37] Cohn J, Schmidt K, Gross R and Ekman P, “Individual differences in facial expression: stability over time, relation to self-reported emotion, and ability to inform person identification", Proc. $4^{\text {th }}$ IEEE Intl. Conf. on Multimodal Interfaces, 2002: $491-496$

[38] Cohen I, Sebe N, Garg A, Chen L and Huang T, "Facial expression recognition from video sequences: temporal and static modelling", Computer Vision and Image Understanding, 91, 2003: 160-187

[39] Dellaert F, Polizin T and Waibel A, "Recognizing Emotion in Speech", Proc Of ICSLP 1996:1970 - 1973

[40] Lee CM, Narayanan S and Pieraccini R, "Recognition of Negative Emotion in the Human Speech Signals", Workshop on Automatic Speech Recognition and Understanding, 2001: 240 - 243

[41] Gorniak P and Roy D, "Probabilistic Grounding of Situated Speech using Plan Recognition and Reference Resolution", $7^{\text {th }}$ Intl. Conf. on Multimodal Interaction (ICMI'05), 2005;138-143

[42] Roy D, Kai-Yuh Hsiao, and Mavridis N, "Mental Imagery for a Conversational Robot", IEEE Trans. Systems, man \& Cybernetics Part B: Cybernetics, 2004, 34(3);1374-1383
[43] Caridakis G, Malatesta L, Kessous L, Amir N, Raouzaiou A and Karpouzis K, "Modelling naturalistic affective states via facial and vocal expressions recognition", Int. Conf on Multimodal Interfaces (ICMI'06), 2006, 9 pages

[44] Balomenos T, Raouzaiou A, Ioannou S, Drosopoulos A, Karpouzis K and Kollias S, "Emotion Analysis in Man-Machine Interaction Systems, Machine Learning for Multimodal Interaction”, Bengio S and Bourlard H (eds.), Lecture Notes in Computer Science, SpringerVerlag 3361, 2004: 318 - 328

[45] Moeslund TB and Granum E, "Special Article: A Survey of Computer Vision-Based Human Motion Capture", Computer Vision and Image Understanding, 2001, 81(3): 231 - 268

[46] Moeslund TB, Hilton A and Krüger V, "Review article: A survey of advances in vision-based human motion capture and analysis", Computer Vision and Image Understanding - Special Issue on Modelling People: Vision-based understanding of a person's shape, appearance, movement and behaviour, 2006, 104(2-3): 90 - 126

[47] Pavlovic VI, Sharma R and Huang TS, "Visual Interpretation of Hand Gestures for Human-Computer Interaction; A Review", IEEE Trans. Pattern Analysis \& Machine Intelligence, 1997, 19(7): 677 - 695

[48] Nguyen Dang Binh, Enokida Shuichi, Toshiaki Ejima, "Real-Time Hand Tracking and Gesture Recognition System”, GVIP 05 Conference, 2005, 7 pages

[49] Xu Zhang, Xiang Chen, Wen-hui Wang, Ji-hai Yang, Lantz V and Kong-qiao Wang, "Hand gesture recognition and virtual game control based on 3D accelerometer and EMG sensors", Proc. $13^{\text {th }}$ Int. Conf. on Intelligent User Interfaces, 2009: 401 - 406

[50] Picard RW, Vyzas E and Healey J, "Toward Machine Emotional Intelligence: Analysis of Affective Physiological State”, IEEE Trans. Pattern Analysis \& Machine Intelligence, 2001, 23(10): 1175 - 1191

[51] Nasoz F, Alvarez K, Lisetti CL and Finkelstein N, "Emotion recognition from physiological signals using wireless sensors for presence technologies", Cognition, Technology \& Work, 2004, 6(1): $4-14$

[52] Penders J, Grundlehner B, Vullers R and Gyselinckx B, "Potential and Challenges of Body Area Networks for Affective Human Computer Interaction", Foundations of Augmented Cognition. Neuroergonomics \& Operational Neuroscience, Lecture Notes in Computer Science, 5638, 2009: 202-211

[53] http://www.newscientist.com/article/dn18350-your-keyboard-knowsthat-its-you-and-youre-stressed.html (last accessed 14 October 2010)

[54] Raptis ME and Dick GN, "The rise of the Silver Surfer: The use of computers and the internet by seniors - an opportunity for continuing education", J. of Information Education Research, 2007, 9(1), 141 161

[55] Selwyn N, "The information aged: A qualitative study of older adults' use of information and communications technology", J. of Aging Studies, 2005, 18(4): 369 - 384

[56] Russell C, Campbell A and Hughes I, "Ageing, social capital and the Internet: Findings from an exploratory study of Australian 'silver surfers"', Australasian Journal on Ageing, 2008, 27(2): 78 - 82

[57] Hebestreit L, "The role of the University of the Third Age in meeting needs of adult learners in Victoria", Australian J. of Adult Learning, 2008, 48(3): $547-565$ 